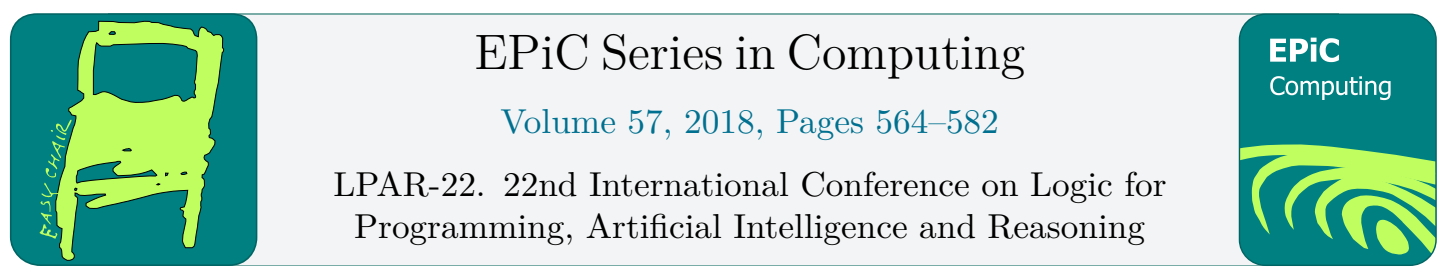

\title{
A Verified Theorem Prover Backend Supported by a Monotonic Library
}

\author{
Vincent Rahli ${ }^{1}$, Liron Cohen $^{2}$, and Mark Bickford ${ }^{2}$ \\ ${ }^{1}$ Luxembourg University, Luxembourg \\ ${ }^{2}$ Cornell University, Ithaca, NY, USA
}

\begin{abstract}
Building a verified proof assistant entails implementing and mechanizing the concept of a library, as well as adding support for standard manipulations on it. In this work we develop such mechanism for the Nuprl proof assistant, and integrate it into the formalization of Nuprl's meta-theory in Coq. We formally verify that standard operations on the library preserve its validity. This is a key property for any interactive theorem prover, since it ensures consistency. Some unique features of Nuprl, such as the presence of undefined abstractions, make the proof of this property nontrivial. Thus, e.g., to achieve monotonicity the semantics of sequents had to be refined. On a broader view, this work provides a backend for a verified version of Nuprl. We use it, in turn, to develop a tool that converts proofs exported from the Nuprl proof assistant into proofs in the Coq formalization of Nuprl's meta-theory, so as to be verified.
\end{abstract}

\section{Introduction}

Interactive theorem provers are remarkable software systems with the aim of mechanizing formal reasoning at the highest level of rigor. Nowadays they are playing a major role in high value applications of formal methods such as certified programming, advanced programming language design, cyber security, cyber physical systems, etc. But what makes a prover trustworthy? There are many different components underlying a theorem prover that must be trusted in order for one to trust the proofs it generates, from the logic it implements down to the hardware on which the code runs. One such key component is the underlying library. In most main theorem provers all the definitions, lemmas, and proofs are stored in a library which underlines the computation system. Such digital libraries allow users to organize, share, search, and reuse their code and knowledge, as well as manage the complexity of large implementations [4]. In this paper we internalize the concept of the underlying library and present a formally verified backend of Nuprl $[19 ; 5]$. Nuprl is a theorem prover that implements a dependent type theory, in the spirit of Martin-Löf's extensional theory [43], which is built on top of an untyped and lazy $\lambda$-calculus.

The first stage in building a formally verified theorem prover was taken in [9] where Nuprl's meta-theory was formalized in Coq [12; 22]. The implementation includes Nuprl's computation and type systems. Nuprl's derivation rules were proven to be valid w.r.t. Allen's PER semantics [3; 2], and the consistency of Nuprl relative to that of Coq's was established. 
In this paper we take the next step by adding support for the dependency of the computation and type systems on the library. This is essential as Nuprl proofs contain computation steps that can unfold user definitions, but also to ensure that standard operations on the library preserves its validity, as we here prove. We thus formalize the concept of a library of interdependent definitions and proofs (where definitions can refer to proofs, and proofs can refer to definitions), and add support for standard manipulations on a library such as creating new definitions and proofs. These operations on the library can be invoked by users through commands that can be combined into a script. Because proofs are built in the context of a library of definitions and lemmas, performing a $\delta$-reduction, i.e., unfolding a definition from the library, counts as a computation step and therefore as a proof step too. In order to be able to verify the validity of proof steps that involve computations, we must have a formal account of what a single computation step is. To support this, we must first extend the computation system with user definitions, which are also called abstractions.

Given that computations, and therefore proofs, depend on the current state of the library, it is essential to prove some preservation properties of the library. The main desired property is that standard actions on a library (e.g. adding new definitions and new lemmas) all preserve its validity. Nuprl has several features that pose some unique challenges in formally verifying such properties of a digital library. In particular, the existence of proof terms (extracts) and undefined abstractions. The main difficulty here is that in any given state of the library most terms, namely the abstractions that have not been defined yet, do not have any operational semantics. However, in the spirit of the open-ended nature of Nuprl [24], we do not want to syntactically forbid the use of undefined abstractions. Moreover, they offer some advantages such as providing a natural way to define (mutually-)recursive functions as illustrated in Sec. 3. Therefore, because undefined abstractions are part of the computation system and may appear in other syntactic objects, we must control their behavior semantically. Objects that mention undefined abstractions are unstable in the sense that they can change their value once these abstractions are defined. Therefore, the meaning of lemmas that mention undefined abstractions does not rely solely on the current library, and hence the semantics must be extended to accommodate the evolving behavior of undefined objects. We here address this issue by first parameterizing the semantics of types and sequents by a library parameter, and then constraining the library parameter so that a sequent is true only if it is true in all possible extensions of the current library (in the spirit of Kripke's semantics [34]).

We prove that this new semantics is a conservative generalization of the old one, in the sense that sound rules w.r.t. the old semantics remain sound w.r.t. the new semantics. Given this new semantics we prove the monotonicity of the system with respect to the library, i.e. that all standard operations on the library preserve the semantics. We also prove a name-invariance theorem, which states that renaming certain entries in the library results in the same theory modulo that renaming. This is a critical feature of the library since in practice we often rename definitions and lemmas in our implementations for various reasons, e.g., to use a different convention, to avoid clashes, etc.

In addition, we build support for using this verified backend implementation as a proof checker for Nuprl in the form of a fully automated tool that takes as input a Nuprl proof and converts it into a proof in the Coq formalization of Nuprl, so as to be checked for validity. It consists of two main components: an OCaml program that translates Nuprl code into Coq code; and a Coq program that verifies the generated Coq code. The tool provides stronger guarantees of validity as it certifies not only the correctness of the logical meta-theory, but also that of a proof kernel for Nuprl. In addition, it can be used as a standalone tool to interactively build Nuprl proofs within Coq, thanks to its extensible command language. 
Outline. The rest of the paper is organized as follows: Sec. 2 surveys some of Nuprl's key features which are essential to the work in this paper. Sec. 3 provides a detailed account of how user definitions are implemented and manipulated in Nuprl. In Sec. 4 we refine the semantics of sequents in Nuprl, which enables the monotonicity and name-invariance properties of the library. Sec. 5 describes the implementation of a library structure in Nuprl. Sec. 6 discusses a fully automated tool, CoqPRL, that we have implemented in order to use our verified proof assistant backend as a proof checker for the current version of Nuprl. Sec. 7 discusses related work, and Sec. 8 concludes.

The entire development presented in this paper has been fully formalized in Coq, and can be accessed here: https://github.com/vrahli/NuprlInCoq. The formalization described here amounts to more than 20K lines of Coq code, on top of the existing formalization of Nuprl in Coq [9] (a precise count is not possible because the additions are interspersed among many files). In addition, the tool CoqPRL described in Sec. 6 amounts to about 4.5K lines of OCaml code.

\section{Background}

Nuprl has a rich type theory, called Constructive Type Theory (CTT), including equality types, a hierarchy of universes, $\mathrm{W}$ types, quotient types [18], set types, union and (dependent) intersection types [33], image types [49], PER types [6], approximation and computational equivalence types [55], and partial types [58; 20;23]. CTT types are interpreted as Partial Equivalence Relations (PERs) on closed terms, by Allen's semantics [3; 2]. CTT differs from other similar constructive type theories, such as the ones implemented by Agda [15; 1], Coq, or Idris $[16 ; 32]$, primarily because it is an extensional type theory (i.e., propositional and definitional equality are identified [28]) with types of partial functions [58; 20;23]. For example, while the fixpoint $\operatorname{fix}(\lambda x . x)$ diverges, it is nonetheless a member of the partial type $\overline{\mathbb{Z}}$, which is the type of integers and diverging terms. ${ }^{1}$ The rich expressiveness of Nuprl renders type checking undecidable, but in practice this is mitigated by type inference and type checking heuristics implemented as tactics.

Nuprl's programming language is an untyped (à la Curry), lazy $\lambda$-calculus with pairs, a fixpoint operator, etc. Fig. 1 presents a subset of Nuprl's syntax and small-step operational semantics [5; 50], which will be used in this paper. A term is either (1) a variable; (2) a value (also called a canonical form) ${ }^{2}$ or (3) a non-canonical term. A non-canonical term $t$ has principal arguments - marked using boxes in Fig. 1-which are terms that have to be evaluated to canonical forms before $t$ can be reduced further. For example the application $f a$, which we often write $f(a)$, diverges if $f$ diverges. The bottom part of Fig. 1 presents part of Nuprl's small-step operational semantics. We omit the rules that reduce principal arguments such as: if $t_{1} \mapsto t_{2}$ then $t_{1}(u) \mapsto t_{2}(u)$. Terms of the form ifpair (another one not shown here is iflam) are called canonical form tests, and are further explained in [55].

Nuprl's types are interpreted as partial equivalence relations (PERs) on closed terms $[3 ; 2$; 23]. The PER semantics can be seen as an inductive-recursive definition of: (1) an inductive relation $T_{1} \equiv T_{2}$ for type equality; (2) a recursive function $a \equiv b \in T$ for equality in a type. For example, one case in the definition of $T_{1} \equiv T_{2}$ is as follows: (i) $T_{1}$ computes to $\Pi x_{1}: A_{1} \cdot B_{1}$; (ii) $T_{2}$ computes to $\Pi x_{2}: A_{2} . B_{2}$; (iii) $A_{1} \equiv A_{2}$; and (iv) for all closed terms $t_{1}, t_{2}$ such that $t_{1} \equiv t_{2} \in A_{1}$, $B_{1}\left[x_{1} \backslash t_{1}\right] \equiv B_{2}\left[x_{2} \backslash t_{2}\right]$. We say that a term $t$ inhabits (or realizes) a type $T$ when $t$ is equal to itself in the PER interpretation of $T$, i.e., $t \equiv t \in T$. The PER interpretation of types entails that an equality type of the form $a=b \in T$ is true (i.e. inhabited by $\star$ ) iff $a \equiv b \in T$ holds. [9; 50].

\footnotetext{
${ }^{1} \overline{\mathbb{Z}}$ can be seen as the integer type of ML-like programming languages like OCaml.

${ }^{2} \mathrm{Nuprl}$ also features exceptions [53; 54], which are omitted here because they are irrelevant to the present discussion.
} 


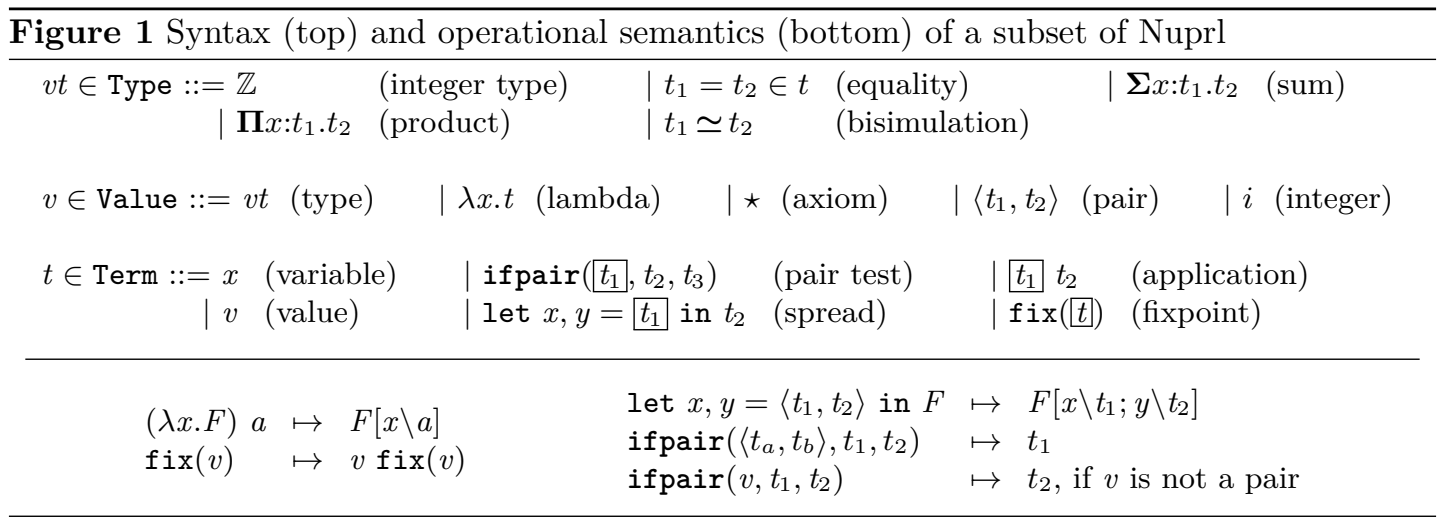

Note that an equality type can only be inhabited by the constant $\star$, i.e., equalities do not have computational content as opposed to the situation in Homotopy type theory [59].

Howe [31] wrote about this PER semantics: "Because of this complexity, many of the Nuprl rules have not been completely verified, and there is a strong barrier to extending and modifying the theory". This is no longer true thanks to the recent formalization of CTT in Coq [9; 50; 53]. The implementation includes: (1) Nuprl's computation system; (2) Howe's computational equivalence relation, and a proof that it is a congruence; (3) a definition of the PER semantics of CTT; (4) definitions of Nuprl's derivation rules, and their soundness proofs w.r.t. the PER semantics $^{3}(5)$ and a proof of Nuprl's consistency. This formalization allows for a safe and mechanical way to verify the soundness of existing as well as new rules. This paper, in turn, builds upon and extends this formalization.

Systems such as Coq, Agda, or Idris are only closed under computation, meaning that one can only substitute a term for another term if they compute to the same value. Nuprl in contrast is closed under the more general notion of Howe's computational equivalence $\sim$, which was proven to be a congruence [29]. In Nuprl, in any context, when $t \sim t^{\prime}$ we can rewrite the term $t$ into $t^{\prime}$ without having to prove anything about types. Therefore, Howe's computational equivalence is commonly used to reduce expressions inside sequents by first proving that terms are computationally equivalent and then using the fact that $\sim$ is a congruence. ${ }^{4}$ To enable that, Nuprl provides the type $t_{1} \simeq t_{2}$, which is the theoretical counterpart of the metatheoretical relation $t_{1} \sim t_{2}$. In general, computing and reasoning about computations in Nuprl involves reasoning about Howe's computational equivalence relation (see, for example, [55] for more details). However, as will be demonstrated in the sequel, the current definition of the relation is not adequate to handle evolving libraries of definitions, and therefore evolving computation systems (see Sec. 4).

\section{Computing With User Definitions}

User definitions in Nuprl are more general than definitions used in other theorem provers such as Coq or HOL, since they include undefined abstractions. In accordance with refinement-based methodologies, Nuprl supports using an abstraction before it is fully defined. The existence

\footnotetext{
${ }^{3}$ See https://github.com/vrahli/NuprlInCoq/blob/master/RULES for a list of Nuprl's inference rules along with pointers to their soundness proofs.

${ }^{4}$ For example, using How's computational computational equivalence, all streams of zeros such as $\mathrm{fix}(\lambda x .\langle 0, x\rangle)$ and $\operatorname{fix}(\lambda x .\langle 0,\langle 0, x\rangle\rangle)$ are computationally equivalent.
} 
of undefined abstractions offers major advantages, such as providing a 'natural' way to define recursive functions. The 'fix' operator, e.g., can be defined using abstractions simply by $\operatorname{fix}(f)==f(\operatorname{fix}(f))$, indicating that the term on the left of the $==$ symbol unfolds to the term on the right. What is more, unconstrained definitions allow for a user-friendly way to support mutual recursion. A user can simply write down the definitions one after the other, as in $A==B$ (which says that $A$ unfolds to $B$ ) followed by $B==A$ (which says that $B$ unfolds to $A$ ), where $B$ is still undefined at the time $A$ is introduced. The unfolding of such definitions behaves precisely as their computational semantics entails.

User definitions in Nuprl are slightly different than those of similar systems such as Coq because their parameters can include some information regarding their binding structures. This is especially useful in untyped languages such as Nuprl because it provides partial information regarding the meaning of the parameters of a definition. This is achieved using generalized variables, which are also called second-order variables in the Nuprl literature.

Example 1. In Nuprl, a list is either a pair of an element and a list, or the constant $\star$, which indicates the end of the list. The map function on lists could be defined as follows:

$$
\operatorname{map}(x . f[x] ; L)==\operatorname{ifpair}(L, \text { let } h, t=L \text { in }\langle f[h], \operatorname{map}(x . f[x] ; t)\rangle, L)
$$

The parameter $L$ is a standard variable, i.e., a generalized variable of arity 0 , while $x . f[x]$ indicates that $f$ is a generalized variable of arity 1, which indicates that map's first argument is meant to be a function. The expression $f[h]$ is the application of the generalized variable $f$ to the term $h$. Note that map occurs in the right-hand-side of the definition, even though it is not yet defined (however, the system does not generate a fixpoint from this definition).

Next, we need to incorporate user definitions into the computation system. In terms of computation, the only relevant fragment of the library is the collection of its definition entries, which we here call a definition library. First, we parameterize Nuprl's small step operational semantics by a definition library. Then, we extend it with a new case that reduces abstractions by looking up the abstraction in the definition library and then substitutes the arguments in the term that it found in the library (we illustrate this mechanism through an example below). To handle user definitions that make use of generalized variables ${ }^{5}$ we extend the substitution mechanism to support generalized substitution, which we illustrate below. ${ }^{6}$

In addition to having the advantage of conveying more information about the binding structure of abstractions, generalized variables also offers the following advantage: without them it is sometimes necessary to instantiate definitions with functions and then $\beta$-reduce some applications, while the same can be performed by a single generalized substitution. We next illustrate this by describing how to reduce the following instance of map: $\operatorname{map}(x \cdot x+1 ;[1,2,3])$.

Retrieval. We first retrieve the generalized term, which we call $T$, that the abstraction maps to in the current definition library, i.e.:

$$
\text { ifpair }(L, \text { let } h, t=L \text { in }\langle f[h], \operatorname{map}(x . f[x] ; t)\rangle, L)
$$

Substitution. The abstraction is turned into a generalized substitution (called sub) by extracting its arguments. Formally, a generalized substitution is a list of triples each consisting of a standard variable, $f$, a list of standard variables, $x_{1}, \cdots, x_{n}$, and a standard term, $t$ (intuitively, $f$ stands

\footnotetext{
${ }^{5}$ A generalized parameter is of the form $x_{1}, \ldots, x_{n} \cdot X\left[x_{1} ; \cdots ; x_{n}\right]$, which indicates that $X$ can be substituted by a term that mentions the variables $x_{1}, \ldots, x_{n}$. We write $X$ for the variable $X[]$.

${ }^{6}$ See sosub's definition in https://github.com/vrahli/NuprlInCoq/blob/master/terms/sovar.v for more details.
} 
for $\left.\lambda x_{1}, \cdots, x_{n} . t\right)$. For the above example, we build the following generalized substitution that essentially maps $f$ to $\lambda x .(x+1)$ and $L$ to $[1,2,3]$.:

$$
[(f,[x], x+1),(L,[],[1,2,3])]
$$

Unfolding. The generalized substitution $s u b$ is then applied to the generalized term $T$ to generate a standard term, i.e. one with no generalized variables. For this we recurse through $T$, until we reach either $f$ or $L$. When we reach the generalized variable $f[h]$, we first apply sub to the list of terms to which $f$ is applied to: here to the singleton list [h], where $h$ is a generalized variable with no subterms. Since $h$ does not occur in $s u b$, this has the effect of building the standard variable $h$ from the generalized variable $h$. Next, we extract the pair $([x], x+1)$ from sub. Then, we build the standard substitution $[(x, h)]$ from the list of variables $[x]$ (the first element of the above pair) and the list of terms $[h]$, and apply it to $x+1$ (the second element of the above pair) to obtain $h+1$. The end result is:

$$
\text { ifpair }([1,2,3] \text {, let } h, t=[1,2,3] \text { in }\langle h+1, \operatorname{map}(x . x+1 ; t)\rangle,[1,2,3])
$$

\section{Semantics}

The generalized notion of user definitions in Nuprl, and in particular the presence of undefined abstractions, while useful in many ways, also poses some challenges in the formal treatment of libraries. This section describes some of these challenges and the ways in which they were solved.

\subsection{Monotonicity}

Extensions by definitions is a well-known methodology that usually forms a monotonic extension of the original logic. That is, by adding new definitions one cannot falsify already established theorems. However, Nuprl's definitions are more general than the 'standard' logical ones, or even the ones used in other theorem provers. Because undefined abstractions are well-formed expressions that can occur in definitions, lemmas, and proofs, one has to be careful when adapting Nuprl's semantics to account for user definitions, in order to avoid the undesirable situation where adding a new definition makes a true lemma false.

The main difficulty is due to the presence of undefined abstractions, Nuprl's computation system, and in turn, Howe's computational equivalence relation, are inherently non-monotonic. The computation system is straightforwardly non-monotonic in the sense that the abstraction foo() does not compute further if it is not defined in the current library, but can reduce further once foo() has been defined. Howe's computational equivalence relation is non-monotonic due to the fact that it equates all expressions that do not compute to values, such as stuck expressions. A stuck expression is one which is not a value but cannot be further reduced, such as 1(0). Another example of a stuck expression, critical to the work here, is an instance of an abstraction that is not defined in the current library. Thus, if $f \circ o()$ and $\operatorname{bar}()$ are two abstractions which are not defined in the library $l i b$, then they are computationally equivalent w.r.t. lib. However, they are not computationally equivalent w.r.t. some extension of the library that defines foo() to be, say, 0 , and bar() to be, say, 1 , because 0 and 1 are not computationally equivalent.

Nuprl's main derivation rule for reasoning about computations relies on Howe's computational equivalence relation. For example, to prove that $((\lambda x . x) 0)$ reduces to 0 , we prove that the proposition $((\lambda x . x) 0) \simeq 0$ is true by invoking the derivation rule which says that $a \simeq b$ is true if $a$ computes to $b$ in one computation step. As mentioned in Sec. 2, in general a type of the 
form $a \simeq b$ is true if the metatheoretical statement $a \sim b$ is true. Therefore, in order to address the above issue, one possible solution is to adapt Howe's computational equivalence relation to make it monotone. However, this would only establish monotonicity at the computation level, and it is unclear whether this will propagate up to the level of the semantics of sequents and rules. Instead, we here take a more direct approach to obtaining monotonicity by adapting the semantics at the level of sequents to account for the dynamic process of extensions by definitions, and most critically, to account for the unstable nature of undefined abstractions. ${ }^{7}$

\subsubsection{Old Sequent Semantics}

Sequents in Nuprl are of the form $h_{1}, \ldots, h_{n} \vdash T$ lext $\left.t\right\rfloor$. The term $t$ is a member of the type $T$, which in this context is called the extract or evidence of $T$. An hypothesis $h$ is a variable declaration of the form $x: A$, where $x$ is a variable and $A$ is a type. Such a sequent states, among other things, that $T$ is a type and $t$ is a member of $T$. A rule is then a pair of a sequent and a list of sequents, which we write as: $\left(S_{1} \wedge \cdots \wedge S_{n}\right) \Rightarrow S$.

Extracts are programs that are computed by the system once a proof is completed using rules such as the standard function introduction rule:

$$
(H, x: A \vdash B\lfloor\operatorname{ext} b\rfloor) \Rightarrow(H \vdash \Pi x: A . B\lfloor\operatorname{ext} \lambda x . b\rfloor)
$$

This rule says that to prove $\Pi x: A$. $B$, it is enough to prove $B$ assuming the extra hypothesis $x: A$. Users do not have to enter extracts; those are automatically computed from the leaves of a proof up to the root of the proof. In the above example, once the system has extracted $b$ for the subgoal, it generates $\lambda x . b$ as extract for the main goal. Once a proof is complete, Nuprl builds an abstraction that points to the extract of the proof. A similar procedure is invoked in systems such as Coq, where lemmas and definitions are interchangeable: once a lemma has been established it can be used as a definition which unfolds to its extract (which in Coq is simply the proof of the lemma).

Several equivalent definitions for the truth of sequents occur in the Nuprl literature $[8 ; 19$; $23 ; 33 ; 9] .{ }^{8}$ Since our results are invariant to the specific semantics, we do not repeat these definitions here. Instead, we take trueSequent to be any of the possible definitions of the truth of a sequent. The sequent semantics standardly induces the notion of validity of a rule of the form $\left(S_{1} \wedge \cdots \wedge S_{n}\right) \Rightarrow S$ in the following way:

$$
\text { (trueSequent } \left.\left(S_{1}\right) \wedge \cdots \wedge \operatorname{trueSequent}\left(S_{n}\right)\right) \rightarrow \operatorname{trueSequent}(S)
$$

\subsubsection{Modified Sequent Semantics}

The library of Nuprl has always had an implicit impact on the semantics of sequents. This is because the sequent semantics is build on top of the PER semantics, which is based on the computation system. In order to unfold abstractions as part of computations, this dependency had to be made explicit. Thus, we first adapt the semantics of types so as to also depend on a library of definitions.

Example 2. Prior to this work, the integer type $\mathbb{Z}$ was interpreted by the following 4-ary INT predicate:

$$
\operatorname{INT}\left(\tau, T, T^{\prime}, \phi\right)=T \Downarrow \mathbb{Z} \wedge T^{\prime} \Downarrow \mathbb{Z} \wedge\left(\forall t, t^{\prime} . t \phi t^{\prime} \Longleftrightarrow \exists i . t \Downarrow i \wedge t^{\prime} \Downarrow i\right)
$$

\footnotetext{
${ }^{7}$ See https://github.com/vrahli/NuprlInCoq/blob/master/per/sequents_lib.v for details.

${ }^{8}$ All these definitions were shown to be equivalent [8, Sec. 5.1]. Our Coq formalization uses a simplified version of Kopylov's definition [33].
} 
This states that $T$ and $T^{\prime}$ are equal types of the type system $\tau$ if they both compute to the integer type $\mathbb{Z}$ ( $a \Downarrow b$ denotes that a computes to $b$ ); and $\phi$ is the partial equivalence relation of the integer type, i.e., $t$ and $t^{\prime}$ are equal members of $\mathbb{Z}$ if they both compute to some integer $i$. The other type constructors were similarly interpreted by 4-ary predicates that described when two types are equal and uniquely defined their PERs.

The new 5-ary interpretation of the integer type is now parameterized by a library:

$$
\operatorname{INT}\left(l i b, \tau, T, T^{\prime}, \phi\right)=T \Downarrow_{l i b} \mathbb{Z} \wedge T^{\prime} \Downarrow_{l i b} \mathbb{Z} \wedge\left(\forall t, t^{\prime} . t \phi t^{\prime} \Longleftrightarrow \exists i . t \Downarrow_{l i b} i \wedge t^{\prime} \Downarrow_{l i b} i\right)
$$

where $a \Downarrow_{l i b} b$ says that a reduces to $b$ w.r.t. the definition library lib.

Consequently, we parameterize the trueSequent predicate by a library of definitions: trueSequent $(S, l i b)$. Then, we define a predicate that captures the notion of monotonicity (i.e. that a true lemma in some library stays true in any extension of the library) as follows:

$$
\text { monTrueSequent }(S, l i b)=\forall l i b^{\prime} \text {.extends }\left(l i b^{\prime}, l i b\right) \rightarrow \operatorname{trueSequent}\left(S, l i b^{\prime}\right)
$$

where extends $\left(l i b^{\prime}, l i b\right)$ says that every abstraction defined in $l i b$ has the same definition in $l i b^{\prime}$. That is, $l i b^{\prime}$ is allowed to have a larger set of abstractions than lib. The induced semantics says that to prove that a sequent is valid, we now have to prove that it is valid in all possible extensions of the library. ${ }^{9}$

Accordingly, the semantics of a rule $R=\left(S_{1} \wedge \cdots \wedge S_{n}\right) \Rightarrow S$ at a given library lib is naturally generalized from:

$\operatorname{trueRule}(R, l i b)=\left(\operatorname{trueSequent}\left(S_{1}, l i b\right) \wedge \cdots \wedge \operatorname{trueSequent}\left(S_{n}, l i b\right)\right) \rightarrow \operatorname{trueSequent}(S, l i b)$

to the following:

monTrueRule $(R, l i b)=$

$\left(\right.$ monTrueSequent $\left.\left(S_{1}, l i b\right) \wedge \cdots \wedge \operatorname{monTrueSequent}\left(S_{n}, l i b\right)\right) \rightarrow \operatorname{monTrueSequent}(S, l i b)$

This semantics states that Nuprl's rules are immune to the system's open-ended nature [30].

It is straightforward to prove that:

Theorem 1 (Conservativity of the new semantics).

$$
(\forall l i b . \operatorname{trueRule}(R, l i b)) \Rightarrow(\forall l i b . \operatorname{monTrueRule}(R, l i b))
$$

Using this result, it is then straightforward to transition the soundness proof of Nuprl to the new monotonic semantics. If the statement of a rule does not mention the library then we can straightforwardly use Thm. 1 to transition the proof of its validity to the new semantics. This was the case for all the rules we have included so far in our verified checker, except one: the rule to unfold abstractions, which mentions the library in the statement of the rule itself. ${ }^{10}$ In that single case, we had to reprove the validity of the rules according to the new semantics. ${ }^{11}$

This shows that properties about the current state of a library that were ever used in Nuprl proofs are always preserved under extensions of the library. The fact that none of the rules had to be changed in order to comply with the new semantics provides further evidence for the adequacy of the modified semantics. This, in turn, enables us to prove the monotonicity property according to which all standard actions on the library (see Sec. 5) preserve its validity.

\footnotetext{
${ }^{9}$ See sequent_true_ext_lib's definition in https://github.com/vrahli/NuprlinCoq/blob/master/per/ sequents_lib.v.

${ }^{10}$ see https ://github.com/vrahli/NuprlInCoq/blob/master/rules/rules_unfold.v.

${ }^{11}$ See https ://github.com/vrahli/NuprlInCoq/blob/master/rules/rules_mon.v for pointers to the rules that we have proved correct w.r.t. the new monotonic semantics. Also, see https://github.com/vrahli/ NuprlInCoq/blob/master/per/weak_consistency.v for a consistency proof under the new semantics, i.e. a proof that False cannot be proved using the semantics.
} 
Theorem 2 (Monotonicity).

$$
\forall l i b, S \text {. monTrueSequent }(S, l i b) \rightarrow \forall l i b^{\prime} \text {.extends }\left(l i b^{\prime}, l i b\right) \rightarrow \operatorname{monTrueSequent}\left(S, l i b^{\prime}\right)
$$

It should be noted that while we chose to modify the semantics at the level of sequents rather than Howe's computational equality, this change to the semantics also percolates down to reasoning about computations. It constrains the theory in such a way that we can only prove that two undefined abstractions are computationally equal if they are syntactically equal. This is because two undefined abstractions could always be defined differently in two different extensions of the library.

Remark 1. The main focus of this work is to allow building up a library of knowledge with the guarantee of preserving validity. Nonetheless, another key property of libraries is conservativity, i.e. that adding new definitions does not increase the provability power over the original library. The presence of undefined abstractions again makes this non-trivial because an abstraction that occurs undefined in a given library can be defined in an extension of that library, thus permitting new results concerning the previously undefined object. Consider the following: Let lib be a library containing the sole definition: $f \circ o()==$ bar () . Clearly, under lib, one cannot prove $f \circ o()=4 \in \mathbb{Z}$. However, extending the library by adding the definition: $\operatorname{bar}()==4$ enables us to prove $f \circ o()=4 \in \mathbb{Z}$. While the example above shows that general extensions by definitions are not conservative, it also hints that the source of non-conservativity is the ability to define an undefined abstraction. If all abstractions are fully 'covered' by the original library, then any extension of the library is conservative. Formally establishing this requires further development, such as formalizing notions of a library covering a sequent and dealing with cyclic definitions, and is so left for further work.

As part of the formal treatment of libraries and undefined abstractions, another key property is name-invariance. By this we mean that renaming some entries in a library results in the same theory modulo the same renaming. This property is essential in any programming or logical paradigm, for example, to allow users to change the names of their definitions and lemmas at any time. In that respect, nominal logic has recently been explored as a convenient framework for handling names $[26 ; 52 ; 51]$. To support renaming, we have implemented a renaming operation, $r$, which is similar to the name-swapping operation in nominal logic. It is straightforward to prove that this renaming operator preserves operations on terms, such as computing the free-variables of a term, or the substitution operation. Formally, we show that Nuprl is name-invariant by proving the following. ${ }^{12}$

Theorem 3 (name-invariance).

$$
\forall r \text { : Renaming. monTrueSequent }(S, l i b) \rightarrow \text { monTrueSequent }(r(S), r(l i b))
$$

\section{$5 \quad$ Library}

Thus far we only considered definition libraries, i.e., libraries whose entries are abstractions. We next provide a more general notion of a library that also contains facts. This section provides an overview of the main concepts and methods of the formal implementation. For the full formalization see: https://github.com/vrahli/NuprlInCoq/blob/master/rules/proof_with_lib_ non_dep.v.

\footnotetext{
${ }^{12}$ See https://github.com/vrahli/NuprlInCoq/blob/master/rules/name_invariance.v for details.
} 


\subsection{Generalized Libraries}

A rigid library is a list of definitions and proofs. A proof entry consists of a proof name name (simply a string in the implementation), and a Nuprl proof prf.

Proofs are inductively defined as trees of instances of proof rules, called proof steps (essentially proof rule names). In the inductive definition of the type of proof steps each proof rule has a constructor. For example, the constructor 'proof_step_hypothesis' takes a variable $x$ as argument, and is used to prove a sequent of the form $G, x: A, J \vdash A\lfloor$ ext $x\rfloor$. The 'proof_step_lemma' constructor differs from the other ones because it does not refer to any specific proof rule. Instead it takes a lemma name as argument, and is used to build a proof from one stored in the current library by referring to the name of the proof.

As a part of our formal treatment of a library, we also implement the concept of a soft library, which is a rigid library augmented by a list of incomplete proofs. Digital libraries often contain incomplete information, and soft libraries are intended to capture such natural behavior of libraries. As opposed to rigid libraries which contain knowledge that cannot be changed, a soft library contains incomplete and evolving knowledge. We sometimes simply call a soft library, a library. As opposed to a proof tree of a fact, an incomplete proof, which we also call a pre-proof, might have holes, i.e., leaves for which no proof tree has been provided yet. Pre-proofs are defined similarly to complete proofs except that (1) sequents, which we call pre-sequents in this context, do not contain extracts - extracts are built only once a proof is complete; and (2) the pre-proof type has an additional constructor for a hole in a proof, which allows one to make a proof hole that can later be filled with another pre-proof, and eventually with an actual complete proof. It is then straightforward to define a partial function that turns incomplete proofs into complete ones whenever possible, i.e., whenever the incomplete proof does not contain further holes.

\subsection{Script}

A script is a sequence of standard operations on the library. We have implemented support for writing scripts instead of directly writing (pre-)proof trees and definition entries. Formally, a script is a list of commands of the following types (the script language can be of course extended to support additional operations):

- Adding a new definition. (Note that redefining abstractions is prohibited.)

- Starting a new proof.

- Updating an incomplete proof at a given address in the tree. If the address points to a hole, then the hole is updated according to the proof step, i.e., replaced by the rule instance corresponding to the proof step.

- Completing a proof by: (1) verifying that there are no more holes in it, (2) building its extract, and (3) committing the proof to the library, i.e., moving it to the rigid part.

- Renaming a user definition throughout the library.

Each command produces an updated library and a list of messages, that among other things report whether or not the command succeeded. We also use those messages to output useful information such as the collection of all the holes in a proof, which is convenient when interactively building proofs. 


\section{$5.3 \quad$ Extracts}

As mentioned in Sec. 4.1.1, Nuprl provides a way to extract programs from proof trees, such that if $p$ is a proof that $T$ is true, then the extract $e$ extracted from $p$ is guaranteed to be a member of $T$. This allows us to build an abstraction in the library for each proven lemma that unfolds to the extract of the proof. The abstraction's name is simply the name of the proof. When building the extract of a proof $p$, if we reach a leaf that corresponds to an already proven lemma $p^{\prime}$, which is stored in the current library, we simply return the corresponding abstraction that unfolds to the extract of $p^{\prime}$.

\subsection{Validity}

A rigid library is called valid if all its entries are valid w.r.t. previous entries. The validity predicate on rigid libraries is recursively defined so that the empty rigid library is valid, and an extension by a new entry is valid only when the entry itself is valid w.r.t. the proof context that consists of the rest of the library. A definition entry is valid w.r.t. a proof context if it does not shadow, i.e. does not redefine, any definition from its context. A proof entry is valid w.r.t. a proof context essentially if: (a) its name does not shadow any definition from its context (a proof is considered as a definition that points to the extract of the proof); (b) it is well-formed, i.e. each of its nodes corresponds to an instance of a valid proof rule, most notably nodes that refer to other proofs have to refer to proofs that are in the context (in which case our Coq formalization guarantees that the root sequent of the proof is true according to Allen's PER semantics). A soft library is called valid if its rigid part is valid, and its incomplete part is valid w.r.t. the rigid part. The validity predicate on soft libraries is defined similarly, with the difference that holes are considered as valid proof steps.

Using the monotonicity property described in Sec. 4.1, we have proven that all the actions of a script preserve the validity of the library to which they are applied. Given that the empty library is clearly valid, this entails that any application of a script results in a valid library. Consequently, our formalization can produce certificates of validity for libraries constructed in this manner.

\section{Proof Checker}

Now that the formal treatment of libraries provides us with the guarantee that standard operations on the library preserve its validity, the next step is to provide a convenient frontend user interface to this backend implementation in order to make this verified version of Nuprl usable. This could either be done by building a new interface to our formalization, or it could be done by using the current version of Nuprl as the frontend.

Regarding the first approach, in addition to building a convenient visual interface, one needs to at least provide support for tactics. This is left for future work. However https://github. $\mathrm{com} / \mathrm{vrahli} /$ NuprlInCoq/blob/master/rules/proof_with_lib_non_dep_example1.v illustrates how our verified theorem prover backend can be used to conduct simple proofs. To this end, in addition to features to extend a library, our formalization supports commands to interactively build proofs, that can display parts of an incomplete proof. In addition, all our commands output a trace of messages that explain whether or not the commands were successfully applied. Understanding why a proof step was not valid is extremely useful to interactively build proofs. We next focus on the second approach by building a tool that uses the Coq formalization of Nuprl's meta-theory to automatically check Nuprl proofs. 


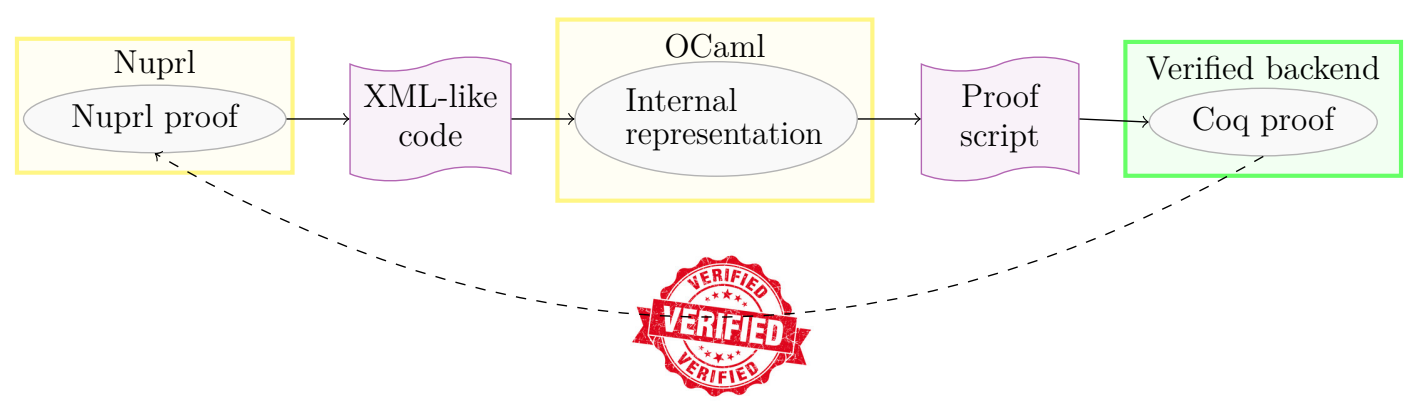

Figure 2: CoqPRL's architecture

\subsection{The CoqPRL Tool}

This section presents CoqPRL, a tool that converts proofs exported from the Nuprl proof assistant into proofs in the Coq formalization of Nuprl's meta-theory, so as to be verified. Note that CoqPRL could also be used in the aforementioned first approach as a way to transfer the vast amount of knowledge that has been accumulated over the years in Nuprl into this new verified theorem prover. This, on its own, seems to be a worthy large scale project for future work.

We next describe the internals of this proof checker. ${ }^{13}$ Those are also illustrated in Figure 2, where we use yellow for the non-certified components (i.e. those that have to be trusted), and green for the certified ones. Given an entry extracted from Nuprl's library, the following steps are taken:

XML-like file. The Nuprl system provides functionalities to export proofs (i.e. entries in the library) to an XML-like format. This is done by first automatically computing all the dependencies of the entry (definitions, proof trees, and proof rules), and exporting those in an XML-like file, which our tool can parse.

Internal representation. Next, we have written an OCaml program that automatically turns the XML-like code into an internal representation, essentially an interleaving list of Nuprl definitions and proof trees. This internal structure facilitates extracting the various parts composing definitions and proofs.

Script. Given an internal representation, CoqPRL then converts it into a script as described in Sec. 5.2. One advantage of using scripts is that they are more compact and thus smaller to store than proof trees.

Verified backend. Finally, scripts are evaluated by our Coq formalization in order to build the corresponding proof within the Coq framework, by applying each command one at a time, and updating the library accordingly, producing validity certificates on demand.

\subsection{An Illustrative Example}

We now provide an example of a library automatically generated using CoqPRL. The code presented here is an exert of the code generated by our tool when given as input a Nuprl proof that True (defined in Nuprl as $0=_{\mathbb{Z}} 0$ ) is a type. See https://github.com/vrahli/NuprlInCoq/

\footnotetext{
${ }^{13}$ See https://github.com/vrahli/NuprlInCoq/blob/master/nuprl_checker/README.md for further details.
} 
blob/master/nuprl_checker/README. md for an explanation on how to generate the code presented in this section. We chose this example because, while it is a simple assertion, proving it relies on different features of Nuprl, most notably: it uses abstractions that need to be unfolded as well as auxiliary lemmas. In the code excerpts presented in the sequel, generated using CoqPRL, keywords are highlighted in red, constructors in maroon, definitions in green, inductive types in blue and variables in purple.

Our tool is set up to generate an alternating list of abstractions and proofs. It starts by generating an empty library.

Definition vlib0 : ValidUpdRes $:=$ initValidUpdRes.

Definition lib0 : SoftLibrary := initSoftLibrary.

where a ValidUpdRes is a triple of (1) a soft library, (2) a list of messages, and (3) a certificate of the validity of the library; lib0 is the initial empty library; and vlib0 is lib0 accompanied by a proof that it is valid.

Then it generates scripts (omitted here) to produce a library containing the definitions and auxiliary lemmas used in the proof that True is a type. Next, it outputs scripts to produce the definition of True and the proof that it is a type:

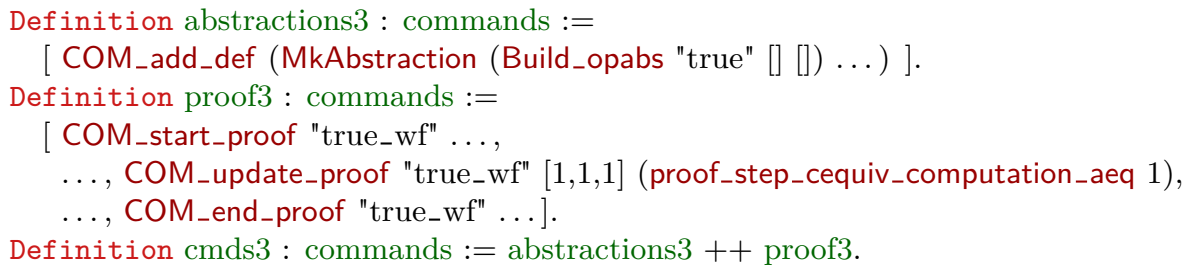

The list of commands abstractions 3 contains a single abstraction called "true", which defines True as $0=_{\mathbb{Z}} 0$. The list of commands proof 3 contains a command to start a new proof called "true_wf", then commands to updates this pre-proof, and finally a command to complete the proof.

Finally, we create the new library lib3 by updating the previous library using the commands in abstractions 3 and proofs3. The "certified" library vlib3 is composed of (1) the library lib3; (2) a proof that it is valid; and (3) a list of messages that indicates here that all commands succeeded.

Definition vlib3 : ValidUpdRes := update_list_with_validity vlib2 cmds3.

Definition lib3 : SoftLibrary $:=$ valid_upd_res_state vlib3.

Evaluating this code and producing such validity proofs makes use of a large part of our $290 \mathrm{~K}$ LOC formalization. For example, proving the validity of the proof makes use of the proofs that Nuprl's proof rules are valid, which relies on the interpretation of Nuprl's types as PERs on closed terms, which in turn relies on Nuprl's computation system. Also proof steps such as proof_step_cequiv_computation_aeq_-which is used to prove that two terms are computationally equivalent [29] - rely on the fact that Nuprl's operational semantics is defined as an executable function.

\subsection{Extensions}

The examples we have explored demonstrate that this automation is feasible, but it remains to develop the mechanism in general since not all of Nuprl's rules are currently supported. There are 262 rules in Nuprl's underlying type theory, and to date 162 of them (which support the core 
type theory) have been formally verified in the Coq formalization. Our tool currently supports 36 of those rules. Nonetheless, our tool is designed to be easily extensible to support additional proof rules. Thus, while tedious, adding support for a new proof rule does not require modifying any of the rest of our $290 \mathrm{~K} \mathrm{LOC}$ formalization. One simply needs to:

1. prove the validity of the rule;

2. add a new constructor to the type of proof steps;

3. express what it means for such a proof step to be well-formed;

4. define how to build an extract for such a proof step;

5. and define how to apply such a proof step in a proof (which in turn ensures that the proof step is well-formed).

It should be noted that to the best of our knowledge, nothing will prevent adding support for the remaining verified and not yet verified rules. Although performance issues should be addressed, preliminary results already indicate that our tool performs reasonably well. For example, the largest example we considered contains 4 abstractions and 355 proof steps ${ }^{14}$. It takes about 15 seconds on a i7-5600U laptop to check the proof in Coq, and about 19 seconds to check the proof and produce a certificate that it is valid. Therefore, we plan to explore ways of improving the running time, e.g. by extracting the Coq code to OCaml.

\section{Related Work}

While to the best of our knowledge our formalization is the first to provide a usable formally verified theorem prover backend for a dependent type theory, several other logical/programming systems have been implemented and verified using proof assistants. Below we discuss some of these systems.

Coq. Barras [10] formalized Werner's [60] set theoretic semantics for a fragment of Coq, which includes the Peano numbers but excludes all other inductive types, in Coq. Werner's semantics assumes the existence of inaccessible cardinals to give denotations to the predicative universes of Coq as sets. In previous work by Barras [11], a deep embedding of a fragment of Coq that excludes universes and inductive types was provided, thereby obtaining a certified typechecker for this fragment. More recently, Anand et al. developed a reifer for Coq terms that can be used to build general purpose plugins, that can be proved correct in Coq, and that can be run efficiently after extraction [7]. In addition, this tool could potentially be used to prove the correctness of Coq in Coq.

HOL Light. In [27] Harrison verified: (1) a weaker version of HOL Light's kernel (with no axiom of infinity) in HOL Light, which is a proof assistant based on classical logic; and (2) HOL Light in a stronger variant of HOL Light obtained by the addition of an axiom that provides a larger universe of sets.

CakeML. Myreen et al. extended the above-mentioned work to build a verified implementation of HOL Light [48; 35; 36] using their verified ML-like programming language - CakeML [37]. CakeML is fully verified in HOL4 down to machine code. In [35] HOL's principles for defining new types and new constants were validated for both a stateless version of HOL, and a stateful

\footnotetext{
${ }^{14}$ To handle larger examples, we will have to add support for more rules using the process described above, which we plan to do over time.
} 
version. The latter was proven correct by translating it into the former. In [36], the correctness of a stateful implementation was directly verified, where sequents depend on a separate "theory". A version of monotonicity for theories was proven, which essentially followed from the fact that they do not allow redefinitions. In this work, in contrast, to obtain monotonicity we had to account for a restricted form of redefinitions, specifically that of instantiating a previously undefined abstraction.

HOL. In [40], the authored improved on previous works [39; 38] and resolved an open problem by establishing conservativity and meta-safety for HOL and HOL based provers over initial HOL. One challenge there was to deal with the intricate interplay between constant and type definitions. In Nuprl there is no notion of extension by type definition, as those are all a part of the initial system (since Nuprl provides a uniform subset type constructor). Moreover, Nuprl's constant definitions slightly differ from that of HOL due to their open-ended nature. Nonetheless, fully formalizing conservativity and meta-safety for constant definitions in Nuprl is, as noted, left for future work.

Milawa. Myreen and Davis formally proved the soundness of the Milawa theorem prover [47] (an ACL2-like theorem prover) which runs on top of their verified Lisp runtime called Jitawa [46].

Buisse and Dybjer. In [17] a categorical model of an intensional Martin-Löf type theory was partially formalized in Agda. As far as we know, this formalization has not led to a verified theorem prover implementation.

KeYmaera $\mathbf{X}$. The core of the KeYmaera $\mathbf{X}$ theorem prover [25] was verified in both Coq and Isabelle in [13]. They verified existing pen-and-paper formalizations and extended the meta-theory to include features used in practice. Moreover, based on these formalizations, they developed verified prover kernels inside those two provers, and left generating verified standalone kernel for future work. More recently, relying on both CakeML and the Isabelle formalization presented in [13], Bohrer et al. introduced VeriPhy, which is a verified pipeline that automatically generates verified controller executables from models that have been verified safe using KeYmaera X [14].

First-order logic. From a formal proof of completeness of first-order logic in Isabelle/HOL, Ridge and Margetson derived an algorithm that can be used to test the validity of formulas [57].

\section{Future Work}

In this paper we implemented the notion of a library, and incorporated it into the formalization of Nuprl in Coq. Nuprl's semantics has been adapted in order to validate the monotonicity of the library, which then allowed us to prove that defining an undefined abstraction preserves the validity of the library We also added support to build up libraries by adding new definitions and proving lemmas. This resulted in a verified backend of (a variant of) Nuprl. Finally, we showed how to use this backend as a proof checker for proofs generated by Nuprl.

An overarching goal of this line of work is turning this verified variant of Nuprl into a full fledged theorem prover. This requires extending the Coq implementation in order to handle all of Nuprl (currently it only supports a portion of it).

Another line of work initiated in this paper, but is yet far from completion, is to formalize and verify other desired library properties such as the ones put forward by Allen et al. in their vision for the behavior of formal libraries [4; 42].

Further work is required in order to turn Nuprl into a completely open-ended system, by allowing for the addition of new computation rules to a library. Currently we have the ability to 
add new terms by defining undefined abstractions, and new proofs. We would like to also be able to add new primitive terms together with their corresponding computation rules.

Abstractions play an important role in the construction of large-scale software and appear in many programming languages. The formal treatment of libraries and systems that can manipulate undefined abstractions presented here opens the door for investigation of several standard programming paradigms, which is left for further study. This includes: incremental programming [21], design-by-contract programming [44; 45; 41] and parametricity [56].

\section{Acknowledgements}

We thank Robert L. Constable and Rich Eaton for their invaluable assistance and helpful criticism. Rahli is supported by the Fonds National de la Recherche Luxembourg (FNR) through PEARL grant FNR/P14/8149128. Cohen is supported by: Fulbright Post-doctoral Scholar program; Weizmann Institute of Science - National Postdoctoral Award program for Advancing Women in Science; Eric and Wendy Schmidt Postdoctoral Award program for Women in Mathematical and Computing Sciences. Bickford is supported by NSF EAGER grant No. 1650069.

\section{References}

[1] Agda Wiki. http://wiki.portal.chalmers.se/agda/pmwiki.php.

[2] Stuart F. Allen. "A Non-Type-Theoretic Definition of Martin-Löf's Types". In: LICS. IEEE Computer Society, 1987, pp. 215-221.

[3] Stuart F. Allen. "A Non-Type-Theoretic Semantics for Type-Theoretic Language". PhD thesis. Cornell University, 1987.

[4] Stuart F. Allen, Mark Bickford, Robert L. Constable, Richard Eaton, Christoph Kreitz, and Lori Lorigo. FDL: A Prototype Formal Digital Library. Tech. rep. 2004-1941. Cornell University, 2002.

[5] Stuart F. Allen, Mark Bickford, Robert L. Constable, Richard Eaton, Christoph Kreitz, Lori Lorigo, and Evan Moran. "Innovations in computational type theory using Nuprl". In: J. Applied Logic 4.4 (2006). http://www.nuprl.org/, pp. 428-469.

[6] Abhishek Anand, Mark Bickford, Robert L. Constable, and Vincent Rahli. "A Type Theory with Partial Equivalence Relations as Types". Presented at TYPES 2014. 2014.

[7] Abhishek Anand, Simon Boulier, Cyril Cohen, Matthieu Sozeau, and Nicolas Tabareau. "Towards Certified Meta-Programming with Typed Template-Coq". In: ITP 2018. Vol. 10895. LNCS. Springer, 2018, pp. 20-39.

[8] Abhishek Anand and Vincent Rahli. Towards a Formally Verified Proof Assistant. Tech. rep. http://www. nuprl.org/html/Nuprl2Coq/. Cornell University, 2014.

[9] Abhishek Anand and Vincent Rahli. "Towards a Formally Verified Proof Assistant". In: ITP 2014. Vol. 8558. LNCS. Springer, 2014, pp. 27-44.

[10] Bruno Barras. "Sets in Coq, Coq in Sets". In: Journal of Formalized Reasoning 3.1 (2010), pp. 29-48.

[11] Bruno Barras and Benjamin Werner. Coq in Coq. Tech. rep. INRIA Rocquencourt, 1997.

[12] Yves Bertot and Pierre Casteran. Interactive Theorem Proving and Program Development. http://www.labri.fr/perso/casteran/CoqArt. SpringerVerlag, 2004. 
[13] Brandon Bohrer, Vincent Rahli, Ivana Vukotic, Marcus Völp, and André Platzer. "Formally verified differential dynamic logic". In: CPP 2017. ACM, 2017, pp. 208-221.

[14] Brandon Bohrer, Yong Kiam Tan, Stefan Mitsch, Magnus O. Myreen, and André Platzer. "VeriPhy: verified controller executables from verified cyber-physical system models". In: PLDI 2018. ACM, 2018, pp. 617-630.

[15] Ana Bove, Peter Dybjer, and Ulf Norell. "A Brief Overview of Agda - A Functional Language with Dependent Types". In: TPHOLs 2009. Vol. 5674. LNCS. http://wiki. portal.chalmers.se/agda/pmwiki.php. Springer, 2009, pp. 73-78.

[16] Edwin Brady. "IDRIS —: systems programming meets full dependent types". In: PLPV 2011. ACM, 2011, pp. 43-54.

[17] Alexandre Buisse and Peter Dybjer. "Towards Formalizing Categorical Models of Type Theory in Type Theory". In: Electr. Notes Theor. Comput. Sci. 196 (2008), pp. 137-151.

[18] Robert L. Constable. "Constructive Mathematics as a Programming Logic I: Some Principles of Theory". In: Fundamentals of Computation Theory. Vol. 158. LNCS. Springer, 1983, pp. $64-77$.

[19] Robert L. Constable, Stuart F. Allen, Mark Bromley, Rance Cleaveland, J. F. Cremer, Robert W. Harper, Douglas J. Howe, Todd B. Knoblock, Nax P. Mendler, Prakash Panangaden, James T. Sasaki, and Scott F. Smith. Implementing mathematics with the Nuprl proof development system. Upper Saddle River, NJ, USA: Prentice-Hall, Inc., 1986.

[20] Robert L. Constable and Scott F. Smith. "Computational Foundations of Basic Recursive Function Theory". In: Theoretical Computer Science 121.1\&2 (1993), pp. 89-112.

[21] William Cook and Jens Palsberg. "A Denotational Semantics of Inheritance and Its Correctness". In: SIGPLAN Not. 24.10 (Sept. 1989), pp. 433-443.

[22] The Coq Proof Assistant. http://coq.inria.fr/.

[23] Karl Crary. "Type-Theoretic Methodology for Practical Programming Languages". PhD thesis. Ithaca, NY: Cornell University, Aug. 1998.

[24] Stuart F. Allen, Robert L. Constable, and Douglas J. Howe. "Reflecting the Open-Ended Computation System of Constructive Type Theory". In: Logic, Algebra and Computation. Vol. F79. NATO ASI Series. Springer-Verlag, 1990, pp. 265-280.

[25] Nathan Fulton, Stefan Mitsch, Jan-David Quesel, Marcus Völp, and André Platzer. "KeYmaera X: An Axiomatic Tactical Theorem Prover for Hybrid Systems". In: CADE-25. Vol. 9195. LNCS. Springer, 2015, pp. 527-538.

[26] Murdoch Gabbay and Andrew M. Pitts. "A New Approach to Abstract Syntax Involving Binders". In: LICS 1999. IEEE Computer Society, 1999, pp. 214-224.

[27] John Harrison. "Towards Self-verification of HOL Light". In: IJCAR 2006. Vol. 4130. LNCS. Springer, 2006, pp. 177-191.

[28] Martin Hofmann. "Extensional concepts in intensional type theory". PhD thesis. University of Edinburgh, 1995.

[29] Douglas J. Howe. "Equality in Lazy Computation Systems". In: LICS 1989. IEEE Computer Society, 1989, pp. 198-203.

[30] Douglas J. Howe. "On Computational Open-Endedness in Martin-Löf's Type Theory". In: LICS '91. IEEE Computer Society, 1991, pp. 162-172. 
[31] Douglas J. Howe. "Semantic Foundations for Embedding HOL in Nuprl". In: Algebraic Methodology and Software Technology. Vol. 1101. LNCS. Berlin: Springer-Verlag, 1996, pp. 85-101.

[32] Idris. http://www.idris-lang.org/.

[33] Alexei Kopylov. "Type Theoretical Foundations for Data Structures, Classes, and Objects". $\mathrm{PhD}$ thesis. Ithaca, NY: Cornell University, 2004.

[34] Saul A. Kripke. "Semantical Considerations on Modal Logic". In: Acta Philosophica Fennica 16.1963 (1963), pp. 83-94.

[35] Ramana Kumar, Rob Arthan, Magnus O. Myreen, and Scott Owens. "HOL with Definitions: Semantics, Soundness, and a Verified Implementation". In: ITP 2014. Vol. 8558. LNCS. Springer, 2014, pp. 308-324.

[36] Ramana Kumar, Rob Arthan, Magnus O. Myreen, and Scott Owens. "Self-Formalisation of Higher-Order Logic - Semantics, Soundness, and a Verified Implementation". In: $J$. Autom. Reasoning 56.3 (2016), pp. 221-259.

[37] Ramana Kumar, Magnus O. Myreen, Michael Norrish, and Scott Owens. "CakeML: a verified implementation of ML". In: POPL'14. ACM, 2014, pp. 179-192.

[38] Ondrej Kuncar and Andrei Popescu. "A Consistent Foundation for Isabelle/HOL". In: ITP 2015. Vol. 9236. LNCS. Springer, 2015, pp. 234-252.

[39] Ondrej Kuncar and Andrei Popescu. "Comprehending Isabelle/HOL's Consistency". In: ESOP 201\%. Vol. 10201. LNCS. Springer, 2017, pp. 724-749.

[40] Ondrej Kuncar and Andrei Popescu. "Safety and conservativity of definitions in HOL and Isabelle/HOL". In: vol. 2. POPL. 2018, 24:1-24:26.

[41] Rustan Leino. "Dafny: An Automatic Program Verifier for Functional Correctness". In: Logic for Programming, Artificial Intelligence, and Reasoning. Springer Berlin Heidelberg, Apr. 2010, pp. 348-370.

[42] Lori Lorigo. "Information Management in the Service of Knowledge and Discovery". PhD thesis. Cornell University, 2006.

[43] Martin-Löf. "Constructive Mathematics and Computer Programming". In: 6th International Congress for Logic, Methodology and Philosophy of Science. Noth-Holland, Amsterdam, 1982, pp. 153-175.

[44] Bertrand Meyer. "Applying "Design by Contract"”. In: IEEE Computer 25.10 (1992), pp. $40-51$.

[45] Bertrand Meyer. Eiffel: the language. Prentice-Hall, Inc., 1992.

[46] Magnus O. Myreen and Jared Davis. "A Verified Runtime for a Verified Theorem Prover". In: ITP 2011. Vol. 6898. LNCS. Springer, 2011, pp. 265-280.

[47] Magnus O. Myreen and Jared Davis. "The Reflective Milawa Theorem Prover Is Sound (Down to the Machine Code That Runs It)". In: ITP 2014. Vol. 8558. LNCS. Springer, 2014, pp. 421-436.

[48] Magnus O. Myreen, Scott Owens, and Ramana Kumar. "Steps towards Verified Implementations of HOL Light". In: ITP'13. Vol. 7998. LNCS. Springer, 2013, pp. 490-495.

[49] Aleksey Nogin and Alexei Kopylov. "Formalizing Type Operations Using the "Image" Type Constructor". In: Electr. Notes Theor. Comput. Sci. 165 (2006), pp. 121-132. 
[50] Nuprl in Coq. https://github.com/vrahli/NuprlInCoq.

[51] Andrew M Pitts. Nominal Sets: Names and Symmetry in Computer Science, volume 57 of Cambridge Tracts in Theoretical Computer Science. 2013.

[52] Andrew M. Pitts. "Nominal Logic: A First Order Theory of Names and Binding". In: TACS 2001. Vol. 2215. LNCS. Springer, 2001, pp. 219-242.

[53] Vincent Rahli and Mark Bickford. "A nominal exploration of intuitionism". In: CPP 2016. Extended version: http : //www . nuprl . org/html/Nuprl2Coq/continuity-long . pdf. ACM, 2016, pp. 130-141.

[54] Vincent Rahli and Mark Bickford. "Validating Brouwer's continuity principle for numbers using named exceptions". In: Mathematical Structures in Computer Science (2017), pp. 149.

[55] Vincent Rahli, Mark Bickford, and Abhishek Anand. "Formal Program Optimization in Nuprl Using Computational Equivalence and Partial Types". In: ITP'13. Vol. 7998. LNCS. Springer, 2013, pp. 261-278.

[56] John C. Reynolds. "Types, Abstraction and Parametric Polymorphism". In: IFIP Congress. 1983, pp. 513-523.

[57] Tom Ridge and James Margetson. "A Mechanically Verified, Sound and Complete Theorem Prover for First Order Logic". In: TPHOLs 2005. Vol. 3603. LNCS. Springer, 2005, pp. 294 309.

[58] Scott F. Smith. "Partial Objects in Type Theory". PhD thesis. Ithaca, NY: Cornell University, 1989.

[59] The Univalent Foundations Program. Homotopy Type Theory: Univalent Foundations of Mathematics. Institute for Advanced Study: http://homotopytypetheory . org/book, 2013.

[60] Benjamin Werner. "Sets in Types, Types in Sets". In: TACS. Vol. 1281. LNCS. Springer, 1997, pp. 530-546. 